\title{
Guardianes del río. Reflexiones \\ sobre lo que hacemos \\ y aprendemos al lado del agua
}

\author{
Patricia Mines \\ pmines@fadu.unl.edu.ar
}

Ricardo Giavedoni

minesgiavedoni@gmail.com
Docentes e investigadores de la Facultad de Arquitectura, Diseño y Urbanismo. Universidad Nacional del Litoral, Argentina.
Integración de la docencia y la extensión /

Intervenciones

RECEPCIÓN: 24/06/16

ACEPTACIÓN FINAL: 10/10/16

\section{Resumen}

"Guardianes del río" nace como un Proyecto de Extensión de Interés Social de la Universidad Nacional del Litoral (UNL, Santa Fe, Argentina), con el objeto de integrar a los alumnos y exalumnos de la Escuela Media O. Rupp de Alto Verde en la identificación, protección y divulgación del patrimonio natural y cultural de ese barrio costero.

En torno de esta propuesta se han creado, a lo largo de más de 5 años, espacios de aprendizaje para alumnos y docentes como prácticas de educación experiencial, prácticas profesionales y voluntariado, se ha motivado la investigación de espacios virtuales colaborativos y la gestión de estrategias de desarrollo sustentable con relación al turismo y habilitado nuevos proyectos de extensión a escala mayor que fueron tema de reflexión en tesis de maestría.

Desde el punto de vista del aporte al currículo universitario, experiencias como esta ofrecen escenarios para construir conocimientos distintos, para formar y sumar subjetividades, para ensayar métodos sobre el hacer, enseñar y aprender, útiles al momento de modelar de manera sustentable nuestro ambiente.

Palabras-clave

- Educación experiencial

- Ambiente

- Sustentabilidad

- Santa Fe

\section{Resumo}

"Guardiões do rio" nasceu como um projeto de extensão de interesse social na Unversidade Nacional del Litoral (UNL, Santa Fe, Argentina), com o objetivo de integrar alunos e ex-alunos da escola de ensino médio O. Rupp de Alto Verde, na identificação, proteção e divulgação do patrimônio natural e cultural desse bairro. Criaram-se em torno desta proposta e ao longo de 5 anos, espaços de aprendizagem para alunos e docentes como práticas de educação experiencial, práticas profissionais e voluntariado, promoveu-se a pesquisa de espaços virtuais colaborativos e a gestão de estratégias de desenvolvimento sustentável vinculadas ao turismo e se autorizaram novos projetos de extensão a maior escala que foram tema de reflexão em dissertações de mestrado.

Como contribuição ao currículo universitário, experiências como esta oferecem cenários para construir conhecimentos diferentes, formar e somar subjetividades, experimentar métodos sobre o fazer, ensinar e aprender, úteis no momento de modelar de forma sustentável o nosso ambiente.

Palavras-chave

- Educação experiencial

- Ambiente

- Sustentabilidade

- Santa Fe
Para citación de este artículo

Mines, P. y Giavedoni, R. (2016). Guardianes del río. Reflexiones sobre lo que hacemos y aprendemos al lado del agua. En Revista +E versión digital, (6), pp. 224-231. Santa Fe, Argentina: Ediciones UNL. 


\section{6}

se trata de revisar la propia matriz

de los saberes disciplinarios instituidos

cuyo pilar maestro común, no es otro

que la fragmentación y el reduccionismo

ecónomo-tecno-científico

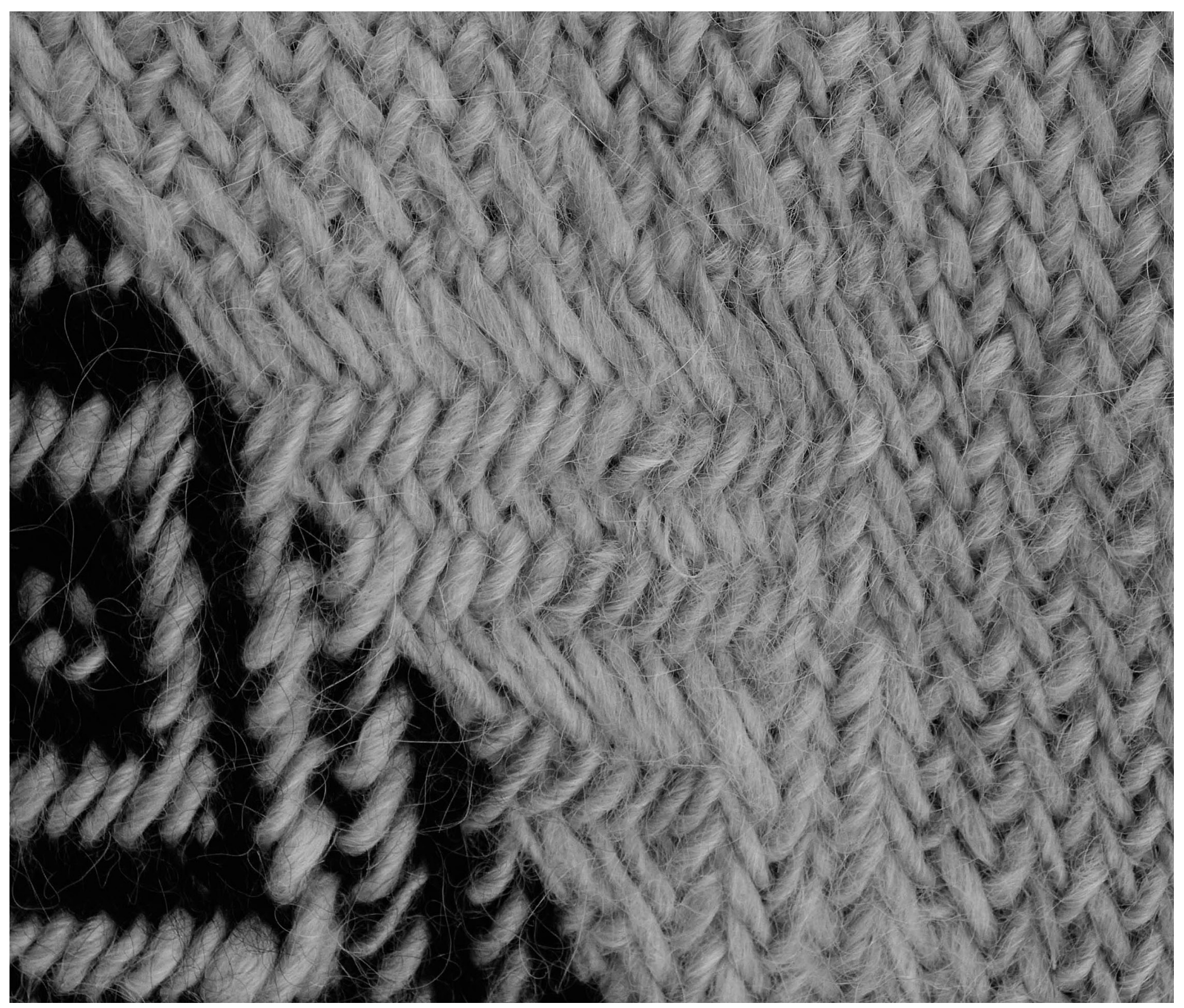

() Micaela Block 


\section{Intervenciones en territorios de agua}

Santa Fe es una ciudad de río. Más del $70 \%$ de la superficie del ejido urbano está constituida por ríos, lagunas y bañados, que forman parte de la rica ecorregión del Delta e Islas del Paraná. Alto Verde se encuentra en medio de estos paisajes. Se trata de un barrio de aproximadamente 18000 habitantes, separado del resto de la ciudad por el riacho Santa Fe. Su origen se remonta a la construcción del Puerto - a inicios del siglo XX-cuando se produjo una de las modificaciones más profundas en el territorio santafecino. A pico, pala y carretilla ${ }^{1}$ se abrió el canal de acceso y rectificó el cauce del río. Con la tierra depositada a un lado, surge Alto Verde, en donde identidad y singularidad de gentes, escenarios paisajísticos, riqueza biológica y vulnerabilidad social e hídrica, son parte del paisaje cotidiano.

Pensar intervenciones que busquen mejorar la calidad de vida en Alto Verde (la ciudad y la región) requiere hacer converger las disciplinas del conocimiento en la identificación de problemáticas y el potenciamiento de su transformación benéfica (Pesci, Perez, Pesci, 2007). Se trata de revisar la propia matriz de los saberes disciplinarios instituidos cuyo pilar maestro común, no es otro que la fragmentación y el reduccionismo ecónomo-tecno-científico (Martínez Díaz, 2010) y abordar el ambiente como sistema de interacciones múltiples (Mihura, 2010) en ciclos proyectuales en donde las sucesivas fases: problema, objetivos, análisis (identificación de conflictos y potencialidades), interpretación sintética sistémica, acción y verificación de la sustentabilidad alcanzada, retroalimentan y enriquecen teoría y práctica. Con esta metodología, el equipo interdisciplinario de docentes, investigadores, graduados y alumnos conformado por arquitectos, biólogos, ingenieros en recursos hídricos, diseñadores gráficos, abogados, especialistas en desarrollo y turismo sustentable, y en ciencias de la educación, ha identificado una variedad y representatividad de conflictos y potencialidades, que ha justificado la elaboración de sucesivos proyectos de extensión. ${ }^{2}$ Algunos de los conflictos más relevantes son la vulnerabilidad socioeconómica ${ }^{3}$ —sobre todo en lo referente a la población joven—, la ocupación indiscriminada de las zonas inundables, la indiferencia a los procesos de crecidas y estiajes, y la degradación ambiental y depredación de los recursos. En tanto que las riquezas naturales, una posición geográfica privilegiada, la singularidad e identidad de su población respecto a su origen, vinculado al puerto y a la vida en las islas, y la credibilidad en las buenas prácticas de los equipos extensionistas universitarios, son parte de las potencialidades detectadas. El primer proyecto "Guardianes del río: jóvenes al cuidado del patrimonio natural y cultural de las islas"4 puso el foco en la identificación de una alternativa de mediación en el problema que plantean las necesidades básicas insatisfechas y la vulnerabilidad de la población joven del barrio Alto Verde, con la necesidad —también básica - de la conservación de los recursos naturales de la rica zona de la eco-región de Islas y Delta del Paraná en donde se encuentra. Trabajamos con los jóvenes alumnos y ex alumnos de la Escuela Media O. Rupp ${ }^{5}$ en la formación de un grupo Guardianes del río, involucrados en el proceso de reconocimiento y construcción del patrimonio de Alto Verde, desde un enfoque integrador.

Un segundo proyecto "Guardianes del río: trabajo sustentable para jóvenes intérpretes del patrimonio de Alto Verde e islas"6 buscó conciliar crecimiento económico, solidaridad social y cuidado de la naturaleza, incluyendo a los Guardianes del río como actores en un territorio más amplio - puerto, zona de influencia, paraje La Boca- en prácticas de turismo sustentable, ensayando
1) Ver http://puertosfe.com/noticias/ 60_Inaugur+el+Museo+del+Puerto+de+Santa+Fe.html

2) Los Proyectos de Extensión de Interés Social (PEIS) son una modalidad de trabajo generada por equipos de extensión de la UNL que abordan diferentes ejes y problemáticas sociales. Los proyectos trabajan en conjunto con organizaciones sociales, gubernamentales y con la comunidad, socializando el conocimiento generado en la institución y procurando concretar acciones transformadoras. A través de convocatorias periódicas, las propuestas son evaluadas y financiadas con presupuesto universitario. En los proyectos participan docentes, graduados, estudiantes y voluntariado como así también los beneficiarios directos de los proyectos, tanto en el diseño como en la ejecución y evaluación de los mismos (http://www. unl.edu.ar/categories/view/cursos\#. WA0ua1ThDIU).

3) En el trabajo "Vulnerabilidad, riesgo y desastres. Sus relaciones de causalidad con la exclusión social en el territorio urbano santafesino", de Arrillaga, Grand y Busso (2009) donde se localiza territorialmente la vulnerabilidad sociolaboral del conglomerado Santa Fe, en la población comprendida en la vecinal Pro-mejoras Alto Verde se manifiesta un índice medio-alto, lo que significa "problemas sociales estructurales, desde necesidades básicas insatisfechas, a insatisfactorios niveles de inserción laboral, con todos las implicancias que ello acarrea".
4) PEIS Guardianes del río: jóvenes al cuidado del patrimonio natural y cultural de las islas (2011-2013)

5) Escuela Técnica Particular Incorporada "Omar Rupp" n 2067, con la Modalidad de Producción de Bienes y Servicios y certificación de Marinero y Auxiliar de Máquinas Navales dependiente del Sindicato de Obreros Marítimos Unidos. Ubicada en la manzana 3 , el número de egresados promedio de los últimos años ronda los 25 alumnos. Su director describe la situación escolar como "urbano-marginal" que posee las características propias de este tipo de escuelas, esto es que los alumnos se encuentran en situación de riesgo sociopedagógico, elevado desgranamiento (en los últimos años, se vio una disminución, creemos que es debido que para el cobro de algunos beneficios sociales se comenzó a exigir la escolarización de los hijos), sumado a esto el alto grado de violencia que suele evidenciarse en este distrito, en donde las discusiones se suelen arreglar con disparos o incluso la muerte, en los últimos años, tuvimos la desgracia que entre alumnos y ex-alumnos sufrimos la muerte de más de 8 de ellos en episodios de violencia. Como resultado de intervenciones anteriores se detectó que los jóvenes expresaban la falta de oportunidades de realización personal y empleo en la zona, y como contracara, la multiplicación de las ocasiones para la delincuencia y el riesgo de caer en adicciones. También se constató que los alumnos, por su pertenencia a un barrio costero de pescadores, 
métodos y técnicas de comunicación estratégica, para revelar el significado de un lugar que es visitado por otros (Morales, Guerra y Serantes, 2009).

La situación de relativo aislamiento de todo Alto Verde se siente de manera extrema en el Paraje La Boca, al sur en la manzana 10, donde el llamado Corte materializa lo antedicho. Se trata de una faja costera de aproximadamente $80 \mathrm{~m}$ de ancho por $2 \mathrm{~km}$ de largo, definida por tierras bajas en tres de sus lados y por el canal de derivación del puerto de Santa Fe en el restante. En el extremo sur del paraje, la escuela Rupp tiene en comodato un terreno en donde está un antiguo Semáforo de Prefectura. Como parte de las actividades de reconocimiento del lugar, se realizaron recorridos por la vecina isla La Tona, en donde los jóvenes alumnos ejercitaron su rol de intérpretes y cuidadosos anfitriones. Así se originó un nuevo proyecto ${ }^{7}$ con la propuesta de construcción de una pequeña infraestructura identificatoria del territorio, en la cual se proponía llevar a cabo actividades de divulgación de patrimonio natural y cultural.

El relato de las actividades y los conocimientos que de allí iban surgiendo alimentaron el desarrollo del trabajo de investigación orientado al diseño de espacios virtuales colaborativos, vinculados a prácticas de extensión, ${ }^{8}$ y dieron marco a las dos jornadas de formación en el Centro de Informática y Diseño de la Facultad de Arquitectura, Diseño y Urbanismo (FADU), en la que los alumnos de la Escuela Rupp trabajaron en el diseño de materiales de difusión sobre el patrimonio de Alto Verde.

Un tercer proyecto "Nuevos territorios. Nuevos consensos. Turismo Sustentable en el Paraje La Boca" "está en desarrollo y encuentra a los Guardianes del río como parte de la oferta turística local. Cinco de los exalumnos capacitados por el proyecto son concesionarios de la embarcación municipal La Ribereña, y en un trabajo conjunto con la Subsecretaría de Turismo de la Municipalidad de Santa Fe llevan adelante paseos náuticos educativo-turísticos desde la estación de embarque del dique 2, Puerto de Santa Fe. Este producto es uno de los itinerarios estratégicos en la Microrregión Insular Santa Fe, Rincón y Leyes, que apunta al desarrollo de turismo sustentable y surge del proyecto de investigación que aborda los procedimientos de gestión del desarrollo sustentable en áreas inundables con gobernabilidad difusa. ${ }^{10}$

\section{Las Prácticas de Educación Experiencial}

Las Prácticas de Extensión de Educación Experiencial (PEEE) ${ }^{11}$ tienen por objeto incorporar las prácticas de extensión a las propuestas curriculares de las carreras de grado, favorecer la creación de espacios de aprendizaje y, en la práctica, de saberes y actitudes para abordar profesionalmente diferentes situaciones sociales.

La PEEE "Un lugar para los guardianes del río" se desarrolló durante 2015 con el formato de Espacio curricular de articulación en el Ciclo medio, nivel 3, sumando a las asignaturas Taller de Proyecto Arquitectónico III (TPAIII), Construcciones II y Urbanismo II de la carrera de Arquitectura de la FADU. Y tuvo como propósitos: - El conocimiento, cuidado y planificación de los frágiles paisajes de La Boca, como vía potente para el desarrollo del paraje, demostrativo de lo que podría ser un desarrollo sustentable para un magnífico Alto Verde.

- La generación de un espacio propio para los "Guardianes del río", la toma de posesión de estos nuevos territorios destinados al desarrollo de actividades vinculadas al reconocimiento y divulgación poseen un alto grado de conocimiento de todo lo relacionado a su entorno natural a lo que se suma el hecho de recibir capacitación técnica en marinería como parte de la currícula escolar. 6) PEIS “Guardianes del río: trabajo sustentable para jóvenes intérpretes del patrimonio de Alto Verde e Islas". 7) Aula virtual proyecto Guardianes del río (https://entornunl.edu.ar/ course/view.php?id=927ovirtual); Diseño de Grupo en red social Facebook: https://web.facebook.com/ groups/851657354874311/?fref=ts; Diseño de Página comunidad en red socia Facebook https://web.facebook.com/ Guardianes-Del-Rio-404493763087813/ en el marco del CAl+D 2011: Diseño, desarrollo y evaluación de espacios virtuales interactivos destinados a actividades de docencia, investigación y extensión en la universidad pública argentina, FADU, UNL. Directora: Mg Arq. María Elena Tosello.

8) $15^{\circ}$ Convocatoria de proyectos de Extensión Universitaria y Vinculación Comunitaria Universidad, Estado y territorio, Subsecretaría de Políticas Universitarias (SPU).

9) PEIS Nuevos consensos. Nuevos territorios. Propuesta de un turismo sustentable en el Paraje La Boca I y II. 10) CAl+D 2011: Procedimientos de Gestión del Desarrollo Sustentable, en Áreas Inundables con Gobernabilidad Difusa. Caso: Microrregión insular Santa Fe, Rincón y Leyes FICH UNL. Director: Mg. Ing. Enrique Mihura. 11) La educación experiencial es definida por Camilloni (2013) como un proceso por el cual una vivencia o un conjunto de vivencias se convierte en "experiencia" y ésta en un aprendizaje reconocido como tal. Cuando el aprendizaje es experiencial, quien aprende lo hace en las condiciones sociales de la utilización auténtica de los conocimientos. Aprende en un marco en el que se apunta al logro simultáneo de fines comunitarios y educativos. El alumno aprende a usa los conocimientos en el trabajo. Las Prácticas de Extensión de Educación Experiencial (PEEE) se enmarcan en el Plan de Desarrollo Institucional de la Secretaría de Extensión de la UNL y tienen por objeto incorporar las prácticas de extensión a las propuestas curriculares de las carreras de grado, favoreciendo la creación de espa- cios de aprendizaje, en la práctica, de saberes y actitudes para abordar profesionalmente diferentes situaciones sociales. Son promovidas por la Dirección de Incorporación curricular de la extensión a través de convocatorias anuales para la presentación voluntaria de Prácticas de Extensión de Educación Experiencial (PEEE) en las unidades académicas (http://www.unl. edu.ar/agenda/index.php?act=showEvento\&id=14004\#. WA0wk1ThDIV). 
del patrimonio natural y cultural de Alto Verde e islas y Alto Verde y puerto.

- El diseño de un lugar que se constituya en una oportunidad para pensar/hacer, en medio de este patrimonio dinámico, y de habitar responsablemente zonas de riesgo hídrico.

Realizada en tres etapas, involucró por parte de la FADU a 6 docentes, 3 tutores y 4 alumnos que participaron de la totalidad de la experiencia; 26 y 37 alumnos en las etapas 2 y 3, respectivamente; 10 alumnos, 3 exalumnos y 2 docentes por la comunidad educativa de la Escuela Rupp; y como parte del equipo de extensión se sumaron docentes Facultad de Ingeniería y Ciencias Hídricas (FICH), docente y alumno de la Facultad de Humanidades y Ciencias (FHUC) y voluntarios de FADU.

La primera etapa fue coordinada por docentes de la cátedra de Urbanismo y tuvo como objetivo el análisis e interpretación del paisaje de La Boca. Comprendió actividades en taller, recorridos por el barrio de Alto Verde (por tierra y por agua), por el paraje La Boca y la isla La Tona. Incluyó registros fotográficos, el relevamiento topocartográfico y el inventario de especies arbóreas en el predio de la Escuela Rupp. Resultado de esta etapa es la planimetría producto del relevamiento, con cotas altimétricas y medidas superpuestas a capturas Google, las fichas de relevamiento de árboles que incluyen ubicación y características de cada especie. Con los materiales y lecturas mencionadas, se inició la segunda etapa, formulada como la Unidad Temática 2 en el Taller de proyecto Arquitectónico III, con modalidad de "esquicio de ideas". En ella se planteó el problema de dar forma a un espacio de encuentro de alumnos y ex alumnos de la Escuela Rupp; divulgación del patrimonio natural y cultural; recepción y acompañamiento de visitantes y futuros ecoturistas. Los objetivos se enfocaron en el desarrollo de las capacidades de interpretación de las necesidades individuales y colectivas; lectura del sitio de implantación y sus cambios, del riesgo hídrico y de la vulnerabilidad social, en la formulación de un pequeño programa de usos, diseño de espacios y utilización de tecnologías adecuadas; el desarrollo de las actitudes necesarias, contemplando dimensiones cognitivas, afectivas y de conducta y aptitudes o destrezas de pensamiento, así como observación e interacción social. Incluyó trabajo en taller y en territorio. En el terreno se realizó una jornada de integración en La Boca, paseo en lancha con los exalumnos entrevistados como informantes, relevamiento fotográfico y confección de croquis con el colectivo Croquiseros Urbanos. ${ }^{12}$ En el taller se contó con la exposición de experiencias similares efectuados por el Taller Valderrama de la Universidad Nacional de Rosario ${ }^{13}$ sobre intervenciones en islas. También se compartió un video producido en el Taller de Medios Audiovisuales, Escuela Mantovani ${ }^{14}$ sobre un fragmento de la rutina de una anciana en su recorrido desde su casa en La Boca al centro de la ciudad. Durante las jornadas en taller se realizaron enchinchadas (puesta en común) y plenario de cierre con exposición de los resultados más significativos. Posteriormente, se confeccionó una planilla de autoevaluación en donde los alumnos pudieron registrar sus niveles de logros en términos de conocimientos, actitudes y destrezas alcanzados en la experiencia a partir de las actividades programas para este fin. Como cierre de esta etapa, se expusieron algunos de los trabajos en el Taller sobre Turismo Sustentable en el paraje La Boca ${ }^{15}$ del que participaron vecinos, alumnos secundarios e instituciones locales y en donde se recibieron opiniones respecto del impacto de la intervención propuesta.

La tercera etapa estuvo a cargo de la cátedra de Construcciones II y se orientó al rediseño bioclimático del espacio de encuentro y/o divulgación (centro de interpretación) y la vivienda del cuidador del predio y culminó en noviembre con excelentes desarrollos. Se abordaron las nociones de arquitectura sustentable/ bioclimática a partir del uso de los materiales, el control climático, la utilización racional de la energía, el uso de patrones locales en las adaptaciones de los habitantes al territorio, los conceptos de modulación y sistematización de la construcción.

La crecida del río hacia fin del cuatrimestre impidió concretar la materialización de alguna de las propuestas, actividad que será retomada en desarrollos posteriores.

\section{Reflexiones en torno al currículo}

Es a partir de un enfoque ambiental integral —mediante confrontación de ideas, búsqueda de nuevos conceptos que den cuenta de las múltiples dimensiones espaciales y temporales de los problemas, generación de capacidades de comprensión grupal e interdisciplinaria, impulso del diálogo de saberes y desarrollo de herramientas y metodologías de intervención- que se podrá operar la síntesis que, junto a una profunda formación humanística
12) Croquiseros Urbanos es un colectivo de personas unidas por pasiones similares: el dibujo a mano alzada y la observación de la ciudad, entendidas como hechos grupales y sociales de nuestro entorno, desde diferentes miradas intentan capturar su dinámica y lo difunden por redes sociales (http://socearq.org/2.0/2015/08/04/ muestra-del-1er-encuentro-nacional-de-croquiseros-urbanos/).

13) Arquitecto Pedro Ferrazini, de la cátedra Introducción a la Arquitectura, AP1 y AP2. Prof. Titular Mg. Arq. Ana Valderrama. FADU. Universidad Nacional de Rosario. 2007-2014. Isla
Charigué. Equipamientos en Centro Cultural Isla del Charigué.

14) Santa: Didi Arese. Realización:

Carla Arese, José Storni. Docentes:

Agustín Falco, Tere Cherry. Taller de

Medios Audiovisuales, 14 min. $10 \mathrm{seg}$

Escuela Provincial de Artes Visuales

Profesor Juan Mantovani, Santa Fe.
15) Taller sobre turismo sustentable en el Paraje La Boca. Noviembre de 2015. PEIS "Nuevos consensos, nuevos territorios. Turismo sustentable en el Paraje La Boca I". UNL. 


\author{
es a partir de un enfoque ambiental \\ integral que se podrá operar la síntesis \\ que, junto a una profunda formación \\ humanística y el sentido social que \\ caracteriza a la educación universitaria \\ argentina, redunde en una mejor calidad \\ de los aportes de nuestra institución, a \\ los problemas comunitarios de nuestro \\ tiempo y entorno cercanos
}

y el sentido social que caracteriza a la educación universitaria argentina, redunde en una mejor calidad de los aportes de nuestra institución, a los problemas comunitarios de nuestro tiempo y entorno cercanos.

De esta manera, la Universidad, comprometida con la búsqueda de soluciones innovadoras a problemas sociales, promoviendo valores democráticos y liderando, con ambos, procesos de desarrollo local-regional, podrá estar a la altura de los desafíos que plantean los problemas contemporáneos.

Pensamos que experiencias como la narrada ofrecen escenarios para ensayar nuevos modos de enseñar y aprender la sustentabilidad en arquitectura ${ }^{16}$, realizando un aporte al currículo universitario -entendido aquí en su sentido amplio: como todo lo que se enseña y todo lo que se aprende en la Universidad- en la construcción de conocimientos distintos (ayudando a definir y redefinir los contenidos que debemos enseñar) y en la identificación del nuevo perfil profesional que la sociedad demanda.

16) Tesis de Maestría en Desarrollo Sustentable. MINES, P. (2014). Cartografía de lo variable. Otra forma de enseñar la sustentabilidad en arquitectura. Tesis de Maestría en Desarrollo Sustentable. UNLa/Flacam. Cátedra UNESCO.

\subsection{Estos nuevos modos, las prácticas de extensión}

Pensamos que para gestionar la sustentabilidad necesitamos buscar modos de identificar y llevar adelante acciones concretas en las intersecciones en donde se cruzan distintos sentidos e intereses. Con estas intenciones, las prácticas de extensión que llevamos adelante se sostuvieron en una metodología orientada a identificar problemas, justificar encuadres, proponer estrategias y diseñar pautas de intervención. Intentamos trabajar sobre problemas complejos, usuarios reales, necesidades concretas, que fueran más allá de objetos disciplinares, en este caso, con la vulnerabilidad de los jóvenes de Alto Verde.

En su desarrollo, los alumnos pudieron proponer y poner a prueba proyectos no convencionales, de experimentación, que usualmente no tienen lugar dentro de los contenidos de las asignaturas.

Esto nos obligó a negociar saberes entre los docentes al interior de la cátedra. Apareció el desconcierto ante situaciones nuevas. $Y$ las diferencias de criterios se hicieron evidentes ante estas propuestas que suscitaron debates sobre la pertinencia disciplinar de algunos proyectos. Al apelar a lo que García Canclini (2010) refiere como saberes predisciplinarios, incorporados en la mirada del arte (en los videos y los croquis), supimos que nos encontrábamos ante las fronteras mismas de la disciplina (entre arquitectura y arte del paisaje).

La fragmentación del currículo se intentó salvar con el espacio de articulación intercátedra (articulación entre asignaturas) en donde 
se acordaron objetos de estudio (territorio con el cual trabajar, programa de necesidades, proyecto base) y se coordinaron cronogramas. Esto requirió coincidencia de temporalidades entre clases teorías y prácticas, entre calendarios académicos y requerimientos sociales, acuerdos entre cátedras de distintas facultades, y la formación de recursos humanos con habilidades en la evaluación de actitudes. Esto resulta bastante trabajoso en un contexto en donde predomina la diversidad de trayectos de los estudiantes y la oferta de múltiples cátedras en el plan de estudios para un mismo ciclo.

Esta puesta en práctica implicó, asimismo, la aceptación de los inconvenientes derivados del clima, las reprogramaciones, reconocimiento de los tiempos de los otros, los "tiempos de la extensión", como dijera una profesora extensionista. Sabemos que una vez que se reconocen estos límites también se abren un montón de oportunidades y se incorpora la incerteza como parte del proceso.

La actividad modeló formas de pensar al involucrar la totalidad de la persona en estas prácticas de implicación en el territorio y conocimiento de sus gentes, abrimos el camino a un pensar y hacer diferente.

En este recorrido, la posibilidad de trabajo interdisciplinario que da el anclaje de los proyectos de extensión, se convierte en espacio privilegiado pero no sencillo de abordar. Si bien la construcción de conocimientos nutridos por la participación y el encuentro de saberes tiene toda una tradición presente en las políticas de extensión, en sus programas y proyectos, no todos los docentes han tenido ocasión de participar en estos procesos, y el momento de trabajar juntos, lleva implícito un desafío muy grande.

\subsection{Desarrollando las capacidades necesarias}

Estos modos de pensar y hacer ayudan a dar forma a un nuevo profesional, ese que requiere la universidad del siglo XXI. Gibbons (1998) señala que las aptitudes básicas que habrán de adquirirse entrañarán la capacidad para usar el conocimiento producido por otros en formas nuevas y generadoras de percepciones distintas. Más que intermediarios, los nuevos profesionales deberán ser mediadores. Kessler (2013) afirma que:

"el mediador transforma, traduce y distorsiona modificando el significado y generando otros nuevos, puede trabajar en múltiples direcciones, incluso algunas contradictorias, desarrollar diferentes estrategias y entender a la especificidad de los entramados con los que opera". (2013:45)
Así, los estudiantes como sujetos sociales - producto y productores de historia y cultura - tendrán, en el marco de las actividades de extensión, la oportunidad de desarrollar capacidad crítica, sensibilidad y profundidad de análisis. Muchas veces, en su disposición a no saber residirá su potencial capacidad de generar nuevos saberes holísticos e integrales, que permitan leer y entender problemáticas sociales y culturales. Podrán desarrollar capacidades para crear más ciudadanía e inclusión social, y adquiriendo autoconfianza, tomar decisiones creativas con autonomía y responsabilidad, conscientes de las necesidades de los otros.

Para Camilloni (2013), el tema es cómo hacemos para pegar el salto de la enseñanza de lo previsible hacia la capacidad para resolver problemas imprevisibles. En este camino exploramos modos de innovación educativa que sirvan de andamiaje en este trayecto. Desarrollando estas destrezas, estaremos enseñando y aprendiendo la sustentabilidad.

\subsection{Qué enseñar y qué no}

Como vimos, la demanda de nuevos conocimientos alentó la búsqueda de otras miradas. Camilloni habla de:

"conocimientos estratégicos, o condicionales que implican saber por qué, dónde, cuándo y cómo se utilizan estos conocimientos, elaborados, particularmente, en relación con situaciones, casos, problemas o proyectos tal y como se encuentran en la vida real". (2013:11)

Viejos y nuevos conocimientos surgieron en el desarrollo los 5 proyectos sobre Alto Verde y el puerto y Alto Verde y las islas y aumentaron la conciencia sobre nuestro territorio. Buscamos generar un saber de relaciones (Morin, 2007), afinando la percepción para descubrir la verdadera trama de la vida (Capra, 1996), en donde las partes y el todo se entiendan en una red de relaciones, y los patrones puedan dar forma al habitar (Pesci, 1990). Uno de los saberes indispensables es el reconocimiento de la matriz natural de nuestra región: la acción de los ríos y los pulsos de inundación representan la principal fuerza que determina y controla nuestra convivencia con el río en todas las escalas. Es necesario articular los conocimientos provenientes de otras disciplinas, como Geografía, Antropología, Ciencias Biológicas, los saberes populares, las tradiciones artísticas y las habilidades políticas, para ir al encuentro del saber en construcción sobre el área metropolitana Santa Fe-Paraná. 
Algunos alumnos reaccionaron ante estos desafíos y aparecieron -entre las respuestas tradicionales con proyectos de arquitecturas abstractos, aisladas del contexto- intervenciones mínimas, respetuosas y comprometidas con el paisaje y su gente. El desafío de legitimar otras miradas disciplinares e incluir otros saberes nos obliga a decidir qué enseñar y qué no enseñar, relegando algunos saberes ya instituidos.

Alumnos y docentes nos lanzamos a esta aventura y decidimos seguir haciendo y aprendiendo al lado del agua.

\section{4}

el desafío de legitimar otras miradas disciplinares e incluir otros saberes nos obliga a decidir qué enseñar y qué no enseñar, relegando algunos saberes ya instituidos.

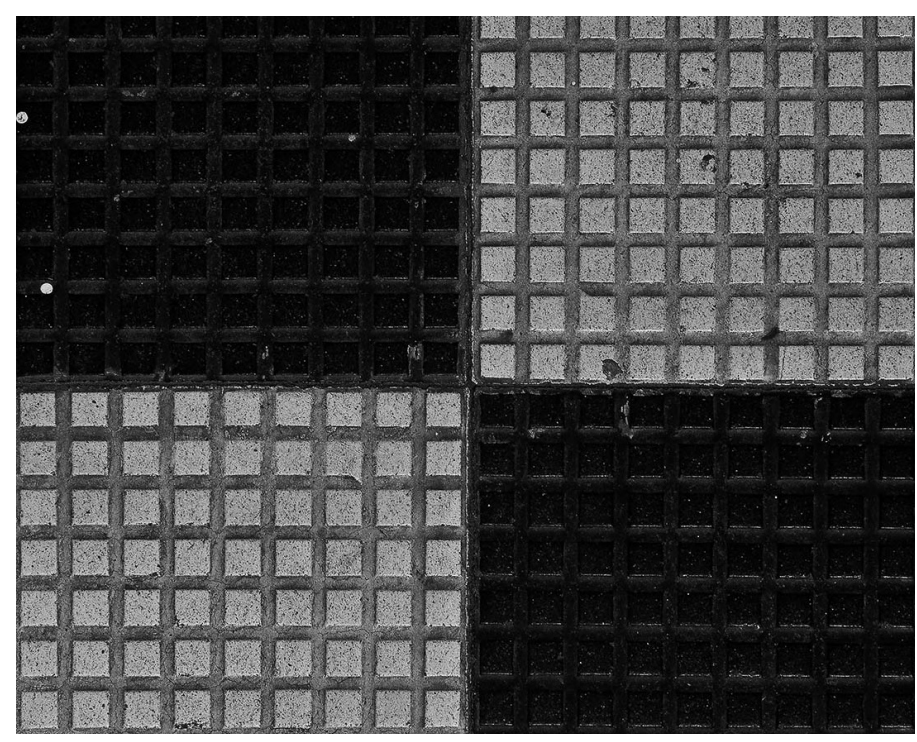

(C) Gastón Pignata

\section{Referencias bibliográficas}

Arrillaga, H.; Grand, M.L. y Busso, G. (2009). Vulnerabilidad, riesgo y desastres. Sus relaciones de causalidad con la exclusión social en el territorio urbano santafesino. En Herzer, H. y Arrillaga, H. (Coords.), La construcción social del riesgo y el desastre en el aglomerado (pp. 59-104). Santa Fe: Ediciones UNL.

Camilloni, A.R.W. de (2013). La inclusión de la educación experiencial en el currículum universitario. En Menendez, G. y otros, Integración docencia y extensión. Otra forma de aprender y de enseñar (pp. 11-21). Santa Fe: Ediciones UNL. Camilloni, A. R. W. de (2012) Curso de Formación La evaluación de los proyectos de extensión incluidos en el currículo. Abril 2012, FADU, UNL. Santa Fe, Argentina.

Capra, F. (1996). La trama de la vida: una nueva perspectiva de los sistemas vivos. Barcelona: Anagrama.

García Canclini, N. (2010). La sociedad sin relato. Antropología y estética de la inminencia. Madrid: Katz Editores.

Gibbons, M. (1998). La pertinencia de la educación superior en el siglo XXI. Conferencia Mundial sobre la Educación Superior de la UNESCO, octubre. París. Kessler, M.E. (2013). Hacia la construcción de una intervención fundada: Ios entramados de la extensión. En Menéndez, G. y otros, Integración docencia y extensión, otra forma de aprender y enseñar. Santa Fe: Ediciones UNL.

Martínez Díaz, G. (2010). Reeducarnos para la sustentabilidad: un intento en la enseñanza de grado en Arquitectura en Educación ambiental en la Universidad de la República. Estado y perspectivas. Grupo de educación ambiental. Red temática de medio ambiente Universidad de la República. Montevideo: Tradinco SA.

Mihura, E. (2010). Reflexiones y aportes para la sustentabilidad de procesos de gestión alternativa de la Educación Superior: estrategias para una Educación para el Desarrollo Sustentable. Tesis Maestría en Desarrollo Sustentable. FLACAMUNLa. Cátedra UNESCO para el Desarrollo Sustentable.

Mines, P. (2014). Cartografía de lo variable. Otra forma de enseñar la sustentabilidad en arquitectura. Tesis de Maestría en Desarrollo Sustentable. UNLa/Flacam. Cátedra UNESCO para el Desarrollo Sustentable.

Morales, J.; Guerra, F. y Serantes, A. (2009). Bases para la definición de competencias en Interpretación del Patrimonio. Fundamentos teóricos y metodológicos para definir las competencias profesionales de los especialistas en Interpretación del Patrimonio en España. CENEAM. www.mma.es/portal/secciones/formacion educacion/grupos_ceneam/interpretacion_patrim onio/pdf/anexo2_bases_competencias_interpr.pdf (recuperado el 29/5/2016).

Morin, E. (2007). Los siete saberes necesarios para la educación del futuro. Buenos Aires: Nueva Visión.

Pesci, R. (1990). La Arquitectura del Ambiente. Ambiente, (66). La Plata: Fondo editorial Cepa.

Pesci, R.; Perez, J. y Pesci, L. (2007). Proyectar la sustentabilidad 2: Enfoque y metodología de FLACAM. La Plata: Fondo editorial Cepa. 